Case Report

\title{
Brain Abscess with Situs Inversus and Dextrocardia: A Case Report
}

\author{
Haroon $\mathrm{K}^{1^{*}}$, Taher $\mathrm{T}^{2}$, Rahman $\mathrm{M}^{1}$, Barua $\mathrm{KK}^{3}$ \\ ${ }^{1}$ Department of Neurosurgery, National Institute of Neuro Science and Hospital, Dhaka, \\ Bangladesh; ${ }^{2}$ Holy Family Red Crescent Medical College Hospital, Dhaka, Bangladesh; \\ ${ }^{3}$ Bangabandhu Sheikh Mujib Medical University, Dhaka, Bangladesh
}

\begin{abstract}
Background: Brain abscess is a neurosurgical emergency. It has to be evacuated without any delay. Though these patients may have congenital heart diseases with ASD and VSD, presence of situs inversus and dextrocardia are relatively uncommon in neurosurgical practice.

Objective: The aim was to present the case with multiple episodes of convulsions and gradual deterioration of consciousness without history of fever.

Methods: The case was carefully evaluated with taking history and examining the patient. Diagnosis was confirmed with a MRI of the brain. It had shown a large ring enhancing lesion in the left temporal lobe which was diagnosed as brain abscess.

Results: The patient underwent left temporal craniotomy and the abscess was totally removed along with the capsule. He made an uneventful recovery.

Conclusion: Dextrocardia and cardiac congenital anomalies are serious conditions especially when they are accompanied with brain abscess. But prompt surgery and careful intraoperative and postoperative care can help the patient to recover early without any adverse event.
\end{abstract}

Keywords: Brain abscess, Dextrocardia, Situs inversus, Craniotomy

\section{Introduction}

Situs inversus total is a congenital condition in which the major visceral organs are reversed or mirrored from their normal positions. This condition generally is an autosomal recessive genetic condition. The incidence of situs inversus total is about $1: 10,000$ live people. ${ }^{1}$

Brain abscess is a serious infection which must be diagnosed early and treated aggressively. ${ }^{2}$ It is an neurosurgical emergency. Predisposing factors identified include congenital heart disease with a right to left shunt, infections of the middle ear, mastoid, paranasal sinuses, orbit, face, scalp, penetrating skull injury, comminuted skull fracture or intracranial surgery including insertion of ventriculo-peritoneal shunts, dermal sinuses and abnormal immune functions. ${ }^{2}$ Diagnosis of a brain abscess is usually made on the basis of neuroimaging findings (eg. CT and MR imaging findings) and clinical examination. ${ }^{3}$

In brain abscess, $20.0 \%$ gram stain and culture positivity were $78.0 \%$. Gram-positive and negative facultative aerobes and obligate anaerobes were also on the rise. Unusual

*Correspondence: Kaisar Haroon, Department of Neurosurgery, National Institute of Neuro Science and Hospital, Dhaka, Bangladesh; e-mail: kaisar298@hotmail.com; ORCID: 0000-0002-3065-7877 organisms, like Burkholderia pseudomallei, Salmonella typhi, Nocardia species, Cladosporium bantiana, Fonsecaea pedrosoi, Entamoeba histolytica, and Acanthamoeba were also isolated and/or detected from the brain abscess aspirate or resected tissue. ${ }^{4}$

\section{The Case}

A 35 year old man presented with the complaints of convulsion three times and decreased level of consciousness. He had history of headache for eleven days and occasional vomiting for the same duration. Suddenly, he had generalized convulsion which had started from the right side of the body and he had lost consciousness. It lasted for few minutes then he was well. But again, he had two bouts of convulsion and he became less responsive. He had no history of fever, sinusitis or ear infection. On examination he was well built. His GCS was $E_{2} V_{2} M_{5}$. Pupils were bilaterally reacting to light. On examination heart sound was on the right side with liver dullness on the left side. His chest X-ray showed dextrocardia with situs inversus (figure 1). His echocardiogram showed dextrocardia with membranous VSD and ASD. His ECG had the features of dextrocardia. His MRI of brain with contrast showed a ring enhancing lesion at the left 
temporeal lobe with perilesional edema, midline shifting and effacemaent of ipsilateral temporal horn (figure 2).

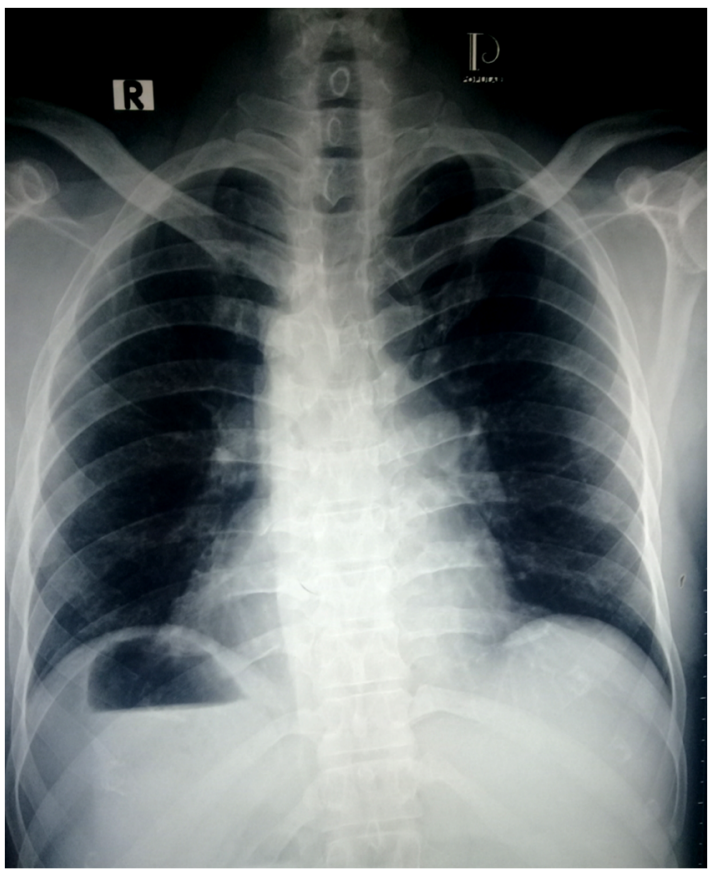

Figure 1: Chest $\mathrm{X}$-ray postero anteriror view showing apex of the heart and fundal gas shadow at the right side.

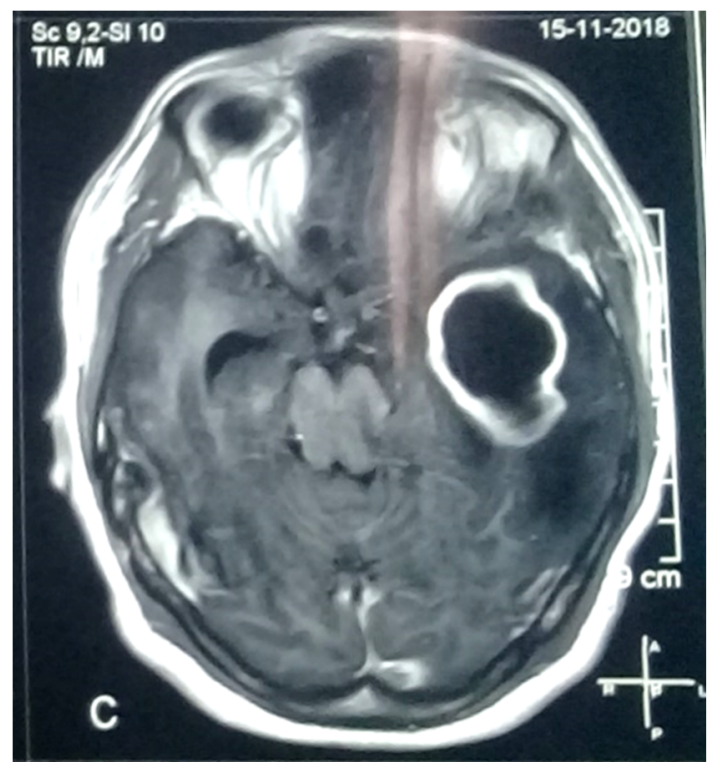

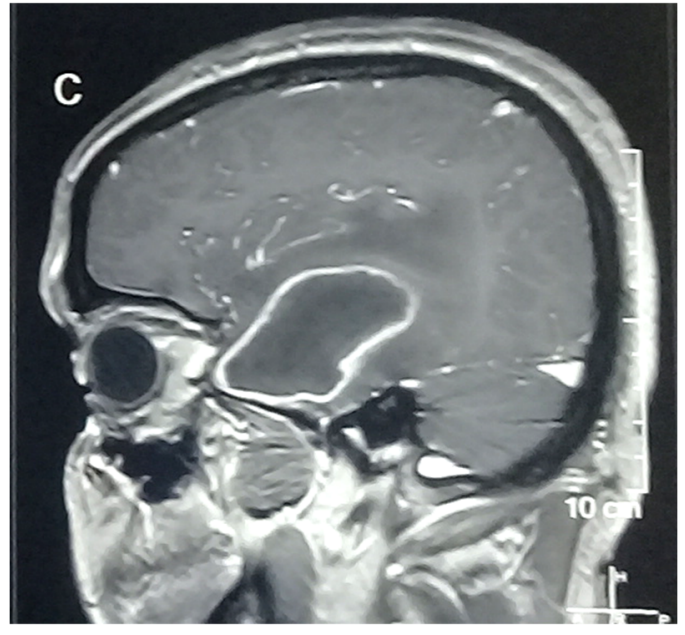

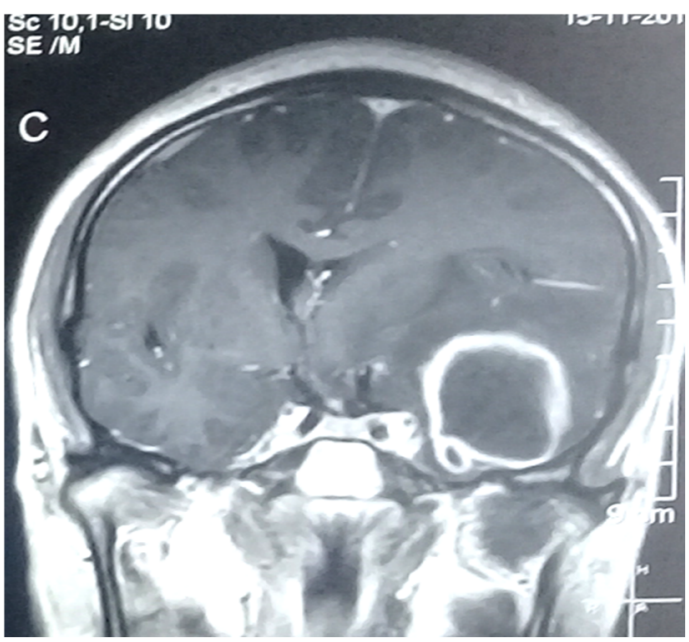

Figure 2: Contrast T1WIMRI of brain showing brain abscess

He had undergone left temporal craniotomy. His brain was very tight. Dura was opened. The abscess was localized and about $48 \mathrm{ml}$ purulent foul smelling pus was evacuated (figure 3). A small corticotomy was done and the pyogenic membrane was totally removed. Haemostsis was achieved with fibrillar surgical and bipolar coagulation. Then dura was closed and wound was closed in layers.

He was kept on triple antibiotic consisting of ceftriaxone, flucloxacillin and metronidazole. His stitches were removed on $8^{\text {th }}$ post operative day and he was discharged. His parenteral antibiotics were continued for two weeks. He continued oral antibiotic for six weeks and made a good recovery without any neurological deficit. 


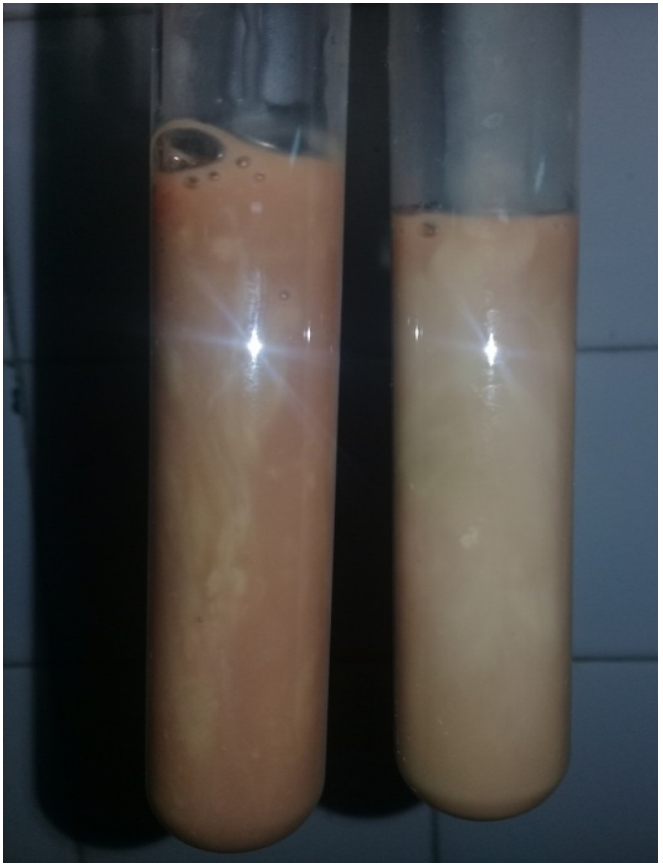

Figure 3: Pus evacuated from the abscess

\section{Discussion}

In congenital heart diseases there is direct shunt of blood from right to the left heart. Venous blood flows directly into the left atrium, it is feasible that emboli can take this pathway to enter the brain. ${ }^{5} \mathrm{Un}$ operated cyanotic congenital heart disease (CCHD) is an important predisposing factor for brain abscess, accounting for $25-46 \%$ of cases. ${ }^{2}$ In our patient there was dextrocardia of the heart and also there was VSD and ASD. This allowed the blood from right heart to go to left heart and then to the brain.

MRI with contrast is an important tool for diagnosing brain abscess. The abscess is a ring enhancing lesion in contrast T1 image and with marked peripheral edema. In MRS: there is increase in lactate, acetate, and succinate presumably originate from the enhanced glycolysis and fermentation of the infecting microorganisms. ${ }^{5-}$ 12 Amino acids such as valine and leucine are known to be the end products of proteolysis by enzymes released by neutrophils in pus. ${ }^{3}$

There are multiple modalities of treatment, ranging from simple burr hole and drainage to craniotomy and total removal of the abscess capsule. In this patient we chose to excise the abscess cavity totally after craniotomy. The capsule was thick and it was relatively accessible after corticotomy. Therefore, it could be easily removed the abscess capsule from the temporal lobe. Burr hole and evacuation results in residual abscess cavity, so there may be recurrence. Total surgical removal of the abscess wall is better than simple burr hole evacuation.

\section{Conclusion}

Dextrocardia and cardiac congenital anomalies are serious conditions especially when they are accompanied with brain abscess. But prompt surgery and careful intraoperative and post operative care can help the patient to recover early without any adverse event.

Conflict of interest: None

Funding: None

Submitted: $16^{\text {th }}$ February, 2020

Final revision received: $17^{\text {th }}$ March, 2020

Accepted: $19^{\text {th }}$ March, 2020

Published online: $1^{\text {st }}$ April, 2020

\section{References}

1. Taher T, Nahar N, Afrin M. Dextrocardia with situs inversus totalis in a 2-months-old boy: A case report. Sir Salimullah Medical College Journal. 2018; 26:112-14.

2. Mehnaz A, Syed AU, Saleem AS, Khalid CN. Clinical features and outcome of cerebral abscess in congenital heart disease. J Ayub Med Coll Abbottabad. 2006;18:21-24.

3. Lai PH, Ho JT, Chen WL, Hsu SS, Wang JS, Pan $\mathrm{HB}$, et al. Brain Abscess and Necrotic Brain Tumor: Discrimination with Proton MR Spectroscopy and Diffusion-Weighted Imaging. American Journal of Neuroradiology. 2002; 23:1369-77.

4. Lakshmi V, Umabala P, Anuradha K, Padmaja K, Padmasree C, Rajesh A, et al. Microbiological spectrum of brain abscess at a tertiary care hospital in South India: 24-year data and review. Pathology Research International. 2011;2011:583139, 12 pages.

DOI: $10.4061 / 2011 / 583139$

5. Frenchu KK, Ahmed MB. Brain abscess in an adult patient with congenital heart disease. JAMA Cardiology. 2019;4:184-85 